TM-1683

[SSCL-N-737]

\title{
Cryostat Design for the Superconducting Super Collider 50mm Aperture Dipole Magnet *
}

\author{
Thomas H. Nicol \\ Fermi National Accelerator Laboratory \\ P.O. Box 500 \\ Batavia, Illinois 60510 \\ Yannis P. Tsavalas \\ General Electric Medical Systems \\ Florence, South Carolina 29501
}

September 1990

* Presented at the 1990 Applied Superconductivity Conference, Snowmass, Colorado, September 24-28, 1990. 


\author{
Thomas H. Nicol \\ Fermi National Accelerator Laboratory \\ P.O. Box 500 \\ Batavia, IL 60510 \\ Yannis P. Travalas \\ General Electric Medical Systems \\ P.O. Box F23 \\ Florence, South Carolina 29501
}

\begin{abstract}
The cryostat of an SSC dipole magnet consists of all magnet components except the cold mass assembly. It serves to support the cold mass accurately and relisbly within the vacuum vessel, provide all required cryogenic piping, and to insulate the cold mass from heat radiated and conducted from the environment. It must function reliably during storage, shipping and handling, normal magnet operation, quenches, and seismic excitations and must be manufacturable at low cost.

The major components of the cryostat are the vacuum vessel, thermal shields, multilayer insulation (MLI) system, cryogenic piping, interconnections, and suspension system. The overall design of a cryostat for superconducting accelerator magnets requires consideration of fluid flow, proper selection of materials for their thermal and structural performance at both ambient and operating temperature, and knowledge of the environment to which the magnets will be subjected over the course their 25 year expected life.
\end{abstract}

This paper describes the design of the current SSC collider dipole magnet cryostat and includes discussions on the thermal, structural, and dynamic considerations involved in the development of each of the major systems.

\section{Introduction}

Fermilab has been involved in the design and production of cryostats for all prototype Superconducting Super Collider (SSC) dipole magnets built since 1986. That work is very well documented in the literature $2-7$. Late in 1989 a fundamental change in the dipole coil design; increasing the physical aperture from $40 \mathrm{~mm}$ to $50 \mathrm{~mm}$, resulted in the need to redesign the dipole cryostat. The larger aperture gave rise to an increase in diameter of the overall cold mass assembly of approximately $25 \%$ and a weight increase of approximately $50 \%$ both of which impact the cryostat design significantly.

Cryostat designs for superconducting magnets are largely driven by thermal and structural considerations. Designers must continually be cognizant of the heat load to the helium system and of the structural loads imposed on the cryostat systems from static weight, shipping and handling, quench loads, and ambient ground motion. These two considerations are generally at odds with one another. Low heat load implies a minimum of structural material conducting heat from the environment. Sound structural design implies material with sufficient strength to resist both static and dynamic forces.

This paper attempts to summarize the results of the design effort to date on the collider dipole cryostat for the $50 \mathrm{~mm}$ magnet. Thermal and structural aspects of the design of each of the major cryostat systems will be described in detail. The $40 \mathrm{~mm}$ dipole cryostat purposely served as the starting point for this design work.
Wherever possible, development work on that and other similar cryostats was borrowed in order to take advantage of proven technology. References to the $40 \mathrm{~mm}$ cryostat will be made for comparison wherever appropriate. Each of the cryostat systems will be addressed in turn; vacuum vessel, $80 \mathrm{~K}$ and $20 \mathrm{~K}$ thermal radiation, multilayer insulation (MLI), cryogenic piping, suspension system, and magnet interconnect.

In order to begin the design process, one needs a good handle on the pertinent allowable heat loads to each thermal station as well as a good definition of the structural environment to which the magnet will be subjected. Table 1 summarizes the thermal and structural design criteria for the $50 \mathrm{~mm}$ dipole which affect the cryostat design ${ }^{1}$. Figure 1 illustrates a cross section through the $50 \mathrm{~mm}$ cryostat.

Table 1. Thermal and Structural Design Criteria

$\begin{array}{lrrr} & 4.5 \mathrm{~K} & 20 \mathrm{~K} & 80 \mathrm{~K} \\ \text { Static Heat Loads } & & & \\ \text { Infrared } & 0.053 \mathrm{~W} & 2.335 \mathrm{~W} & 19.1 \mathrm{~W} \\ \text { Support conduction } & 0.160 \mathrm{~W} & 2.400 \mathrm{~W} & 15.8 \mathrm{~W} \\ \text { Interconnect } & 0.150 \mathrm{~W} & 0.320 \mathrm{~W} & \frac{2.1 \mathrm{~W}}{37.0 \mathrm{~W}} \\ \text { Total static } & 0.363 \mathrm{~W} & 5.055 \mathrm{~W} & 3.0 \mathrm{~W}\end{array}$

Dynamic Heat Loads

Synchrotron rad

Splice IR heating

Beam microwave

Beam gas

Total dynamic

\begin{tabular}{lll}
$2.169 \mathrm{~W}$ & & \\
$0.140 \mathrm{~W}$ & & \\
$0.195 \mathrm{~W}$ & \\
$0.136 \mathrm{~W}$ & & \\
\hline $2.640 \mathrm{~W}$ & & \\
$3.003 \mathrm{~W}$ & $5.055 \mathrm{~W} \quad 37.0 \mathrm{~W}$
\end{tabular}

Structural load summary

Cold mass weight:

Shipping and handling:

$11,360 \mathrm{~kg}$

$2.0 \mathrm{~g}$ vertical

$1.5 \mathrm{~g}$ axial

$1.0 \mathrm{~g}$ lateral

\section{Yacuum Vessel}

The vacuum vessel is the outermost cryostat component and, as such, serves to contain the insulating vacuum. In addition, it functions as the major structural element to which all other systems are ultimately attached to the accelerator tunnel floor. Furthermore, it serves as a pressure containment vessel in the event of a failure in an internal cryogen line. The vessel is 685.5 $\mathrm{mm}$ OD, $7.92 \mathrm{~mm}$ wall with an overall length of 14922.5 $\mathrm{mm}$. Its $40 \mathrm{~mm}$ counterpart was $609.6 \mathrm{~mm}$ OD, $6.35 \mathrm{~mm}$ wall and had an overall length of $16306.8 \mathrm{~mm}$.

The vacuum vessel has five penetrations at which the internal cold mass supports are attached. The internal supports are positioned to minimize the 


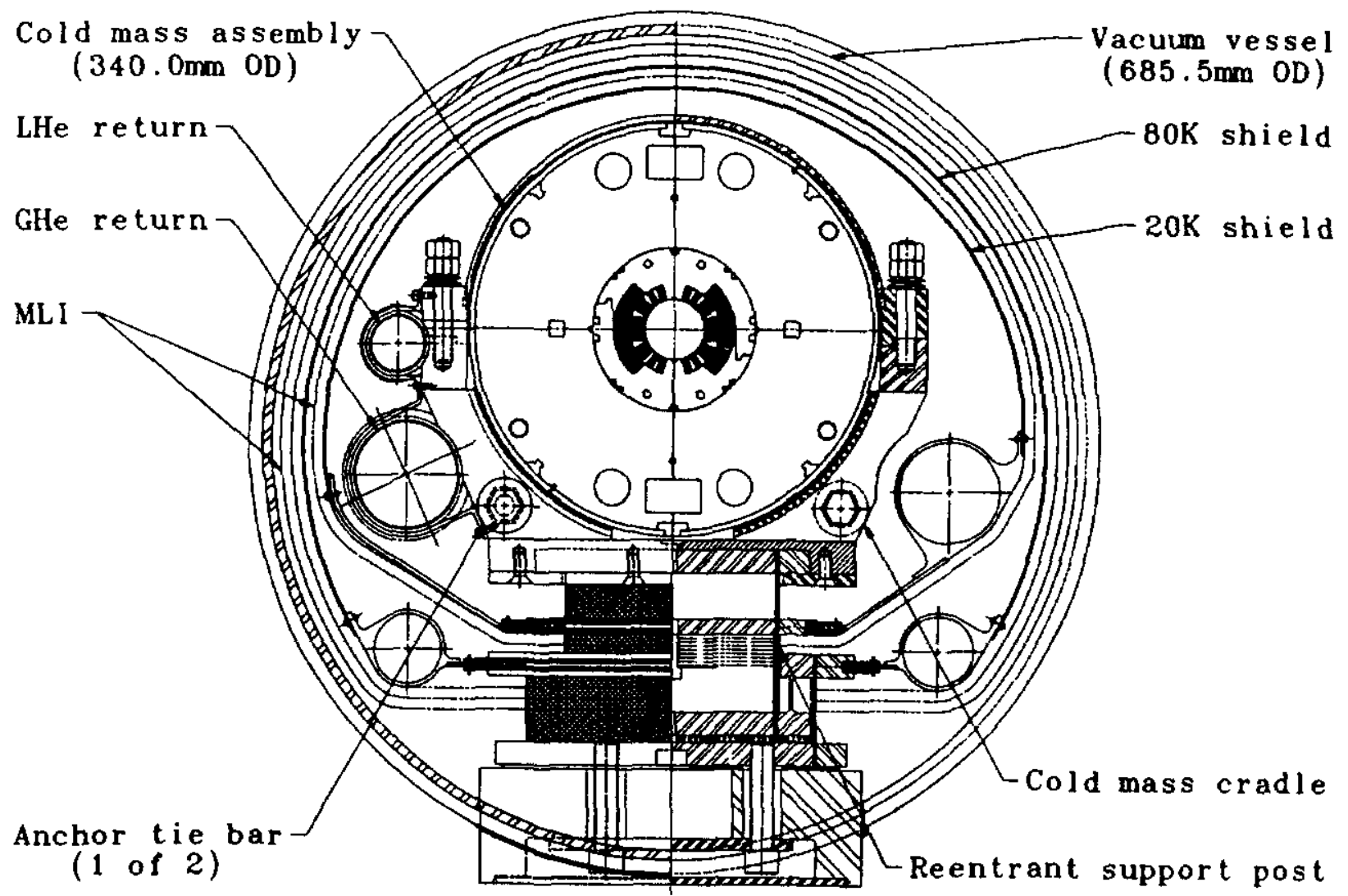

Fig. 1. $50 \mathrm{~mm}$ collider dipole cryostat cross section

deflection of the cold assembly due to its own weight and are spaced on $3179.76 \mathrm{~mm}$ centers starting at the center of the magnet. Two external supports serve to tie the vessel to the tunnel floor. The external supports are spaced to minimize the deflection of the vacuum vessel due to its own weight and to the weight of the internal components. They are located $4154.17 \mathrm{~mm}$ on either side of center.

The $7.92 \mathrm{~mm}$ wall of the vessel is not sufficient to transfer suspension system loads to ground immediately around the internal support locations. At these positions a $22.22 \mathrm{~mm}$ thick, $559 \mathrm{~mm}$ long reinforcement transfers internally generated loads, primarily from shipping and handling, from the magnet support system to the vacuum vessel. The vacuum vessel material is low carbon steel. Ordinarily one would like to use a material with greater fracture toughness in the event of a major cryogen spill inside the cryostat, however, cost mandated a more readily available material. In addition, failure analysis indicated that a high fracture toughness material was not required.

\section{Thermal Radiation Shields}

Like its $40 \mathrm{~mm}$ counterpart, the $50 \mathrm{~mm}$ dipole cryostat uses two thermal radiation shields. The outermost shield is cooled by $\mathrm{LN}_{2}$ and operates at $80 \mathrm{~K}$. The innermost shield is cooled by helium gas returning to the refrigerator and operates nominally at $20 \mathrm{~K}$.

Each shield is cooled by discrete pipes connected to formed shells which totally encompass the internal cold assembly. The $20 \mathrm{~K}$ shield uses a single extrusion attached to the shell. The 80K shield uses two; one serving as the shield supply, the other as the return. This scheme requires that the shells have high thermal conductivity to minimize thermal gradients around their circumference. Copper and aluminum are the materials of choice for this application. Thermal analyses on both shields were performed using both materials. The results from these analyses indicate there is no significant difference in the thermal performance of either shield between these two materials. Material cost dictated the ultimate material choice. Copper and aluminum are approximately the same cost per unit weight, however, with a density over three times that of aluminum, the use of copper as the shield material would result in an assembly cost three times higher than an aluminum shield. The selected material for both $80 \mathrm{~K}$ and $20 \mathrm{~K}$ shields is 6061-T6 aluminum, $1.59 \mathrm{~mm}$ thick. The shields are segmented along their length to minimize the effect of bowing during cooldown caused by the asymmetry of the cooling tubes with respect to the shield shells.

\section{Multilayer Insulation}

The design requirements for the thermal insulation system in the SSC dipole cryostat dictate that heat load from thermal radiation and residual gas conduction be limited to the values listed in Table 1. Essential to meeting these requirements is an insulation system design that addresses transient conditions through high layer density for improved gas conduction shielding, has sufficient mass and heat capacity to reduce the effects of thermal transients, and is comprised of materials suitable for extended use in a high radiation environment. Finally, the system design must be such that fabrication and installation techniques guarantee consistent thermal performance throughout the entire accelerator.

The insulation system must have a mean apparent thermal conductivity of $0.76 \times 10^{-6} \mathrm{~W} / \mathrm{cm}-\mathrm{K}$ in order to 
meet the design heat load budget. This is achieved by using a multilayer insulation (MLI) system comprised of reflective layers of aluminized polyester separated by layers of spunbonded polyester spacer. The reflective layers consist of flat polyester film aluminized on both sides to a nominal thickness not less than 350 angstroms. The spacer layers consist of randomly-oriented spunbonded polyester fiber mats. The mean apparent thermal conductivity of an MLI blanket comprised of these materials has been measured to be $0.52 \times 10^{-6} \mathrm{~W} / \mathrm{cm}-\mathrm{K} 6,7$, The MII system for the SSC $50 \mathrm{~mm}$ collider dipole cryostat will consist of full cryostat length assemblies of MLI fabricated and installed as blankets on the $80 \mathrm{~K}$ and $20 \mathrm{~K}$ shields.

The MLI system for the $80 \mathrm{~K}$ thermal shield consists of two 32-reflective-layer blanket assemblies, for a total of 64 reflective layers. The stack height of each 32-layer blanket is $8.86 \mathrm{~mm}$, with a mean layer density of 3.61 layers per mm. The blanket design incorporates 32 reflective layers of double-aluminized polyethylene terepthalate (PET) film. The reflective layers are separated by single spacer layers of $0.10 \mathrm{~mm}$ spunbonded PET material. Single layers of $0.23 \mathrm{~mm}$ spunbonded PET cover the blanket top and bottom and serve to position the polyester hook and loop fasteners at the blanket edges. The fasteners are affixed to the cover layers by sewing. A third $0.23 \mathrm{~mm}$ PET layer is located midway through the blanket assembly and separates the upper and lower 16 reflective layers of MLI. The multiple blanket layers are sewn together as an assembly along both edges of the blanket. Non-lubricated polyester thread is used in all sewing operations.

The MLI system for the 20K thermal shield consists of two 16-reflective-layer blanket assemblies, for a total of 32 reflective layers. The blankets are designated as the inner $20 \mathrm{~K}$ and outer $20 \mathrm{~K}$ blanket, respectively. The basic design geometry and component materials for the $20 \mathrm{~K}$ MLI blankets are the same as for the $80 \mathrm{~K}$ blankets; the principle differences being the overall width of each blanket and the sewn seam configuration. The blanket design incorporates 16 reflective layers of doublealuminized PET film separated by single spacer layers of $0.10 \mathrm{~mm}$ spunbonded PET material. Single layers of 0.23 mm spunbonded PET cover the blanket top and bottom and serve to position the polyester hook and loop fasteners at the blanket edges. The fasteners are affixed to the cover layers by sewing. The multiple blanket layers are sewn together as an assembly along both edges of the blanket. The 20K blankets are sewn straight through from the upper cover layer to the bottom cover layer.

\section{Cryogenic Piping}

In addition to providing the necessary structural support and thermal insulation for the cold mass assembly, the cryostat serves to contain the piping for all of the cryogenic services required for magnet and magnet system operation. Table 2 provides a summary of the pressure and flow parameters for each of these services. Table 3 lists the pipe sizes for each of the cryostat pipes. Each pipe is anchored at the center of the magnet assembly, and is free to slide axially at the remaining support points to allow for thermal contraction during cooldown.

\section{Suspension System}

The suspension system in any superconducting magnet serves as the structural attachment for all cryostat systems to the vacuum vessel which in turn anchors them to the accelerator tunnel floor.

Conventional suspension systems were effectively reinvented during early SSC magnet development 2,3,5 The intention in development of the $50 \mathrm{~mm}$ dipole cryostat was to take advantage of that earlier work tailoring it only for the revised thermal and structural design parameters. Schedule requirements for the redesign work did not allow continued suspension $R \& D$ and, in fact, the thermal and structural performance of the $40 \mathrm{~mm}$ design did not indicate it to be necessary.

Table 2. Cryogenic System Pressure and Flow Parameters

\begin{tabular}{|c|c|c|c|c|c|c|c|}
\hline System Pr & & uid & $\begin{array}{l}\text { Poper } \\
\text { (atm) }\end{array}$ & $\begin{array}{r}v \\
n 3 / g)\end{array}$ & $\begin{array}{r}\mathrm{T} \\
(\mathrm{K})\end{array}$ & $\begin{array}{r}\text { Flow } \\
(\mathrm{g} / \mathrm{s})\end{array}$ & $\begin{array}{r}\Delta \mathrm{P} \\
(\mathrm{psi})\end{array}$ \\
\hline shld & 18 & $\mathrm{He}$ & 2.5 & 163.4 & $\mathbf{\infty}$ & 100 & 0.016 \\
\hline hld & 18 & $\mathrm{LN}_{2}$ & 5.0 & 1.286 & 84 & 750 & 0.012 \\
\hline Single phs & 18 & $\mathrm{He}$ & 4.0 & 7.255 & 4.25 & 100 & 0.010 \\
\hline LHe & 18 & $\mathrm{H}$ & 3.0 & 7.609 & 4.425 & 85.6 & 0.010 \\
\hline $\mathrm{GHe}$ & 9 & $\mathrm{H}$ & 0.9 & 73.07 & 4.3 & 26.8 & 0.00053 \\
\hline $\mathrm{m}$ & 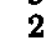 & $\mathrm{H}$ & 0.0 & na & 300 & na & 0.0 \\
\hline
\end{tabular}

Table 3. Cryostat Pipe Sizes (mm)

$\begin{array}{lrrrr}\text { System } & \text { Pipe ID } & \text { Pipe OD } & \text { Flow Area } & \text { Material } \\ & & & & \\ \text { 20K shield } & 82.55 & 88.90 & 5352 & \text { Alum } \\ \text { 80K shield } & 57.15 & 63.50 & 2565 \times 2 & \text { Alum } \\ \text { LHe return } & 45.14 & 47.62 & 1600 & \text { Stn stl } \\ \text { GHe return } & 86.41 & 88.90 & 5864 & \text { Stn stl }\end{array}$

Using the thermal and structural parameters outlined in Table 1 the suspension system for the new design was extrapolated from its $40 \mathrm{~mm}$ counterpart. The emphasis was on meeting the allowed suspension system conduction heat load, satisfying the structural requirements, and maximizing the lateral suspension stiffness. This latter constraint is not explicitly defined in the design criteria, but arose out of concerns during testing of $40 \mathrm{~mm}$ prototypes that the lateral natural frequency was too closely coupled with resonances found in an over the road shipping environment.

The suspension system consists of two major components; reentrant style support posts and anchor tie bars. There are five support posts located along the length of an SSC cold mass assembly spaced $3179.76 \mathrm{~mm}$ apart centered about the middle of the cold mass. Five being the number which limits the cold mass sag due to self weight to the allowed $0.25 \mathrm{~mm}$. The support posts resist vertical and lateral loads imposed during shipping and handling. The cold mass is allowed to slide axially with respect to all but the center post to allow for thermal contraction of the cold assembly during cooldown. This implies that, given no other restraint, the center support would need to resist axial loads. A single support is not capable of resisting this potential $1.5 \mathrm{~g}$ load. Using a special support at the center which could handle this load would impose inordinately high heat loads on the refrigeration system. Rather, a means was developed to distribute axial loads to all five supports without impacting the conduction heat load. Axial tie bars are used to connect the top of each support post to its neighbor(s). In this scheme, an axial load acts first at the center support and then is distributed to all five supports. 
The tie bars must be dimensionally stable when cooled from their assembly temperature of $300 \mathrm{~K}$ to their operating temperature of $4.5 \mathrm{~K}$, otherwise their contraction would impose high bending loads on the support posts. Uniaxial graphite fibers in an epoxy matrix provides the solution. This material is extremely stiff and yet exhibits virtually no change in length during cooldown. An elastic modulus of $82.5 \mathrm{GPa}$ can be achieved in such a composite even with relatively low modulus fibers.

The outer composite tube is $2.16 \mathrm{~mm}$ thick and is filament wound using S-glass in an epoxy matrix. The inner tube is $3.18 \mathrm{~mm}$ thick and is filament wound using graphite fibers in an epoxy matrix. The ability of this design to use two different materials allows each material to be used in its optimum temperature range.

\section{Interconnect}

As its name implies the magnet interconnect serves as the connection area between magnets at which each of the cold mass and cryostat pipes between magnets are connected. Each pipe is anchored axially at the center of the magnet which means they contract between approximately $25 \mathrm{~mm}$ and $32 \mathrm{~mm}$ depending on whether they are stainless steel or aluminum. The relative contraction at the interconnect is then $50 \mathrm{~mm}$ to $64 \mathrm{~mm}$, i.e. twice the single magnet value. Bellows are required on each cold mass, shield, and cryogenic pipe to allow for this expansion. All bellows are hydroformed stainless steel. For the aluminum extrusions on the $80 \mathrm{~K}$ and $20 \mathrm{~K}$ shield pipes an aluminum to stainless steel transition joint is required for the bellows connection. Joints using diffusion bonding or brazing between these two materials have been successfully employed in $40 \mathrm{~mm}$ prototypes. Lateral instability is a concern for interconnect bellows and requires the use of internal squirm protectors on each bellows.

Radiative heat transfer must also be minimized in the interconnect area. This is accomplished by shield bridges which span the $80 \mathrm{~K}$ and $20 \mathrm{~K}$ shield gap between adjacent magnets. These bridges are little more than extensions to the magnet shields, modified to contain their respective bellow $\mathrm{OD}^{\prime} \mathrm{s}$ if necessary. A sliding joint between bridge sections on adjacent magnets accommodates contraction during cooldown. Each is covered with the same MLI scheme used throughout the body of the magnet. These shield bridges also contain pressure reliefs for each shield to prevent pressure buildup within either shield in the event of an internal piping failure. The reliefs are located in the upper half of the shield sections in order to prevent liquid spills from impinging directly onto the vacuum vessel wall.

\section{Summary}

The SSC development program has afforded us the opportunity to extend the design of cryostats for superconducting magnets far beyond the state of the art present at the end of the Fermilab Tevatron program. Advances in new materials technology have opened up options for cryostat designers in both thermal and structural materials. Strict limits on allowable heat load have forced us to develop new mechanisms for structural support and thermal shielding. The end result is a cryostat design which meets the demands of the SSC and which will serve as the starting point for the development of other magnet systems far into the future.
The authors would like to respectfully acknowledge the contributions of W. Boroski, T. Heger, R. Richardson, and $\mathrm{D}$. Arnold without whose help this work would not have been possible.

\section{References}

1. Superconducting Super Collider Laboratory, “15 Meter Collider Dipole Magnet Prime Development Specification," March 6, 1990.

2. Nicol, T.H., et al., "A Suspension System for Superconducting Super Collider Magnets," Preceedings of the Eleventh International Cryogenic Engineering Conference, Vol. 11, Butterworths, Surrey, UK, 1986, pp. $533-636$.

3. Nicol, T.H., et al., "SSC Magnet Cryostat Suspension System Design," Advances in Cryogenic Engineering, Vol. 33, Plenum Press, New York, 1987 pp. $227-234$.

4. Niemann, R.C., et al., "Superconducting Super Collider Second Generation Dipole Magnet Cryostat Design," IEEE Transactions on Magnetics, Vol. 25, No. 2, March 1989, pp. 1615 - 1619.

5. Nicol, T.H., J.D. Gonczy and R.C. Niemann, "Design and Analysis of the SSC Dipole Magnet Suspension System," Super Collider 1, Vol. 1, Plenum Press, New York, 1989, pp. 637 - 649.

6. Boroski, W.N., J.D. Gonczy and R.C. Niemann, Thermal Performance Measurement of a 100 Percent Polyester MLI System for the Superconducting Super Collider Part I: Instrumentation and Experimental Preparation (300K-80K)," Advances in Cryogenic Engineering, Vol. 35, Plenum Press, New York, 1990, pp.487 - 496 .

7. Gonczy, J.D., W.N. Boroski, and R.C. Niemann, "Thermal Performance Measurement of a 100 Percent Polyester MLI System for the Superconducting Super Collider Part II: Laboratory Results (300K-80K)," Adyances in Cryogenic Engineering, Vol. 35, Plenum Press, New York, 1990, pp. 497 - 506. 